\title{
ARE ATHLETES WRONG ABOUT ANABOLIC STEROIDS?
}

\author{
G. R. HERVEY
}

Department of Physiology, The University, Leeds LS2 9JT

It must be one of the oldest of biological observations that males are usually larger and stronger than females. The connection between the testes and male characteristics has been known since remote times, and it is now appreciated that the hormones of the gonads are immediately responsible for most of the visible differences between the sexes. It was thus no surprise when in the 1930s it was reported that testosterone had an "anabolic" action in laboratory animals, causing nitrogen to be retained and body protein, including muscle, to be built up. It was also claimed that in certain synthetic derivatives, the "anabolic steroids", this action had been isolated from the other actions of testosterone. These drugs are not much used in medicine, but in the last decade have been increasingly taken by athletes. This has naturally led to concern, on the grounds of sporting ethics and probable harmful effects. Little effort, however, has been made to find out precisely what effects the substances do produce, and whether these even correspond with the athletes' expectations.

The work of the 1930s, on which the concept of anabolic action was based, has not stood up to critical re-examination. The animals' weight records do not justify the conclusion that sustained weight gain can be produced, too much reliance was placed on urine nitrogen (nitrogen balances are notoriously difficult, and measurements of excretion have led to clearly wrong conclusions in other contexts such as pregnancy and progesterone treatment) and carcass analysis, the only certain test of anabolism, was hardly ever reported. The "rat levator ani" assay deserves a place in history, both for the extent to which it was used as criterion of anabolic activity, and for the remarkable logic behind it. This muscle was selected as representative because out of a number tried, it alone showed increase of weight. Despite the intention to distinguish anabolic from androgenic activity, the chosen muscle was not a skeletal muscle (or homologous with the human levator ani), but a smooth muscle of the rat's reproductive tract, also known as dorsal bulbocavernosus.

In more recent studies in rats (Hervey and Hutchinson 1972) adequate pre-treatment periods were included, treatment was continued long enough for body weight to stablize at a new level, and accurate carcass analyses were done. Administration of testosterone or its derivatives in physiological doses - as judged by restoration of e.g. seminal vesicle weight after castration - did not change body weight, and in larger doses reduced it. This applied to both sexes, with and without gonads and in all circumstances investigated. The "levator ani" showed a similar dose-response pattern to the seminal vesicles, and in contrast to the skeletal muscles which reflected the weight of the whole body. The question "Why then are males larger?" was answered by the finding that in the rat testosterone acting at about the time of birth irreversibly changes the pattern of subsequent growth from female to male pattern (Harvey, Hervey and Hutchinson 1972). This resembles the early determination of sex differences in hormone control and behaviour, and effects of early nutrition. Steroids in general play an important part in controlling body weight and composition (Hervey 1969), and oestrogens do affect adult body weight by an action exerted during adult life and capable of reversal or re-initiation; the action of testosterone, however, appears to be early in life and once for all.

In the present investigation an attempt was first made at a clinical type of enquiry, including any athletes who in medical confidence would give a history of steroidtaking. This revealed interesting beliefs and attitudes, but no factual data. It evidently is widely believed that anabolic steroids increase strength and performance, par- $\frac{\mathbb{D}}{\triangle}$ ticularly in "heavy" events such as shot-putting, and also $\overrightarrow{\vec{P}}$ that their introduction led to a visible upturn in graphs recording performance standards over the years (Payne 1975).* The primary effect is not, however, seen as increase of weight. This may occur, particularly if the training regime includes over-eating, but the more positive consensus is that, if steroids are taken during training, fatigue is retarded and the work done per session increased: the additional training is thought to provide the real benefit. This belief poses athletes with a dilem. ma. Should they take steroids, against sporting conscience and medical advice; or should they refrain, thereby (they believe) forfeiting the chance of reaching the top?

The athletes' conviction that the drugs are effective raises an intriguing scientific problem, and there is clearly a desire on their part to know the true position. It may also be hoped that the administrative problem of controlling steroid-taking will be assisted by better un- $\stackrel{O}{C}$ derstanding. A controlled human experiment was there- $\mathbb{\mathscr { D }}$ fore designed and carried out by Hervey, Knibbs, Hutchinson, Jones, Burkinshaw, Norgan, Hayter and Adamson.

* My impression from his carefully compiled graphs was that, although, as he confirms, athletes have this belief, it is not really supported by the evidence.

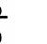


The subjects were 13 men aged 19-25 taking Physical Education in a teacher-training course; they volunteered after a full explanation of the purpose and procedures of the experiment. A double-blind, cross-over design was used ( 11 subjects completed this and the other 2 added an unmatched pair). There were two treatment periods of six weeks during which each subject took steroids, or an outwardly indistinguishable placebo, and followed a standard daily weight-training routine. The subjects had not in general engaged in weight-training prior to the experiment. The order of treatment was determined randomly. The two periods were separated by five weeks of holiday without training. On the basis that it should be typical of what athletes actually take, the steroid treatment was made $100 \mathrm{mg} /$ day of methandrostenolone (Dianabol), given orally as $5 \mathrm{mg}$ tablets.

The primary aim was to study changes in the amounts of the components of the body by as many methods as possible. In addition to simple weighing, these included total body potassium measured by the natural radioisotope ${ }^{40} \mathrm{~K}$ in a whole-body counter; body water by tritium dilution; lean:fat ratio from densitometry by underwater weighing; body fat from skinfold thickness; muscle and subcutaneous fat thickness by roentgenogrammetry. Secondly, athletic performance was measured by leg, arm and trunk work in weight-training; static leg and hand strength by dynamometry; impulsive work by a force-platform jump; and maximum oxygen consumption on a cycle ergometer. Thirdly, studies were made of steroid metabolism. Some checks were made of Dianabol excretion by Professor R. V. Brooks. The subjects were regularly examined clinically and blood pressure and plasma concentrations of cholesterol, bilirubin and the enzymes GPT and GOT measured.

In the illustrations which follow, the thin lines connect measurements at the beginning and end of the two experimental periods for the 11 subjects who completed both. The heavy lines join the means; the differences given are those between the mean changes over the two periods; the significance tests compare the changes over the periods as paired data.

Figure 1 shows the changes in body weight. There was a substantial and consistent increase over the period on the drug. This averaged $3.4 \mathrm{~kg}$, and was significantly different from the negligible change over the period on the placebo.

For the purposes of body composition studies the body is often regarded as made up of two components, the lean body and fat, each of constant and known composition, combined in varying proportions. The lean body includes cells, body fluids and supporting structures such as bone; fat is the pure lipid. On this basis the body potassium, which the ${ }^{40} \mathrm{~K}$ method measures with an accuracy of about $4 \%$, is a measure of the lean body;
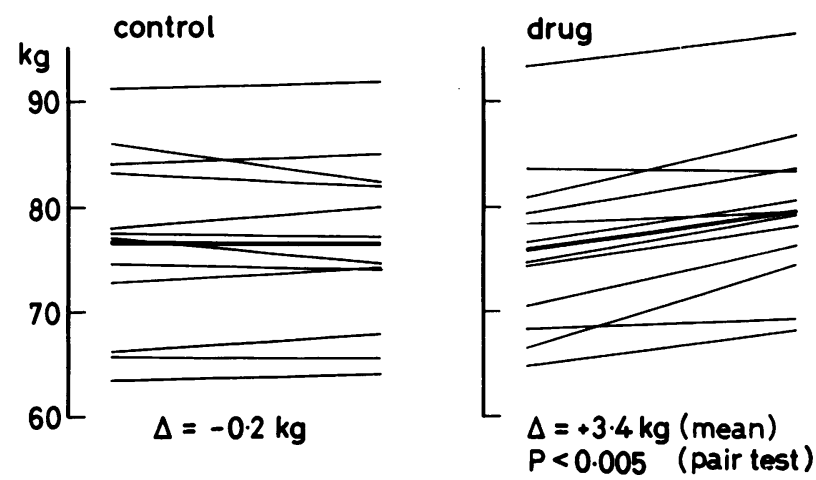

Fig. 1. Changes in body weight. In this and the following figures the thin lines connect measurements made at the start and end of the experimental periods; the period on the placebo is shown on the left, and on methandrostenolone on the right; for the 11 subjects who completed both periods. The heavy lines connect the mean values. $\Delta$ gives the difference between the mean changes on drug and placebo; the value of $P$ gives the significance of this, tested by a t-test, taking the changes in individual subjects over the two periods as paired data.
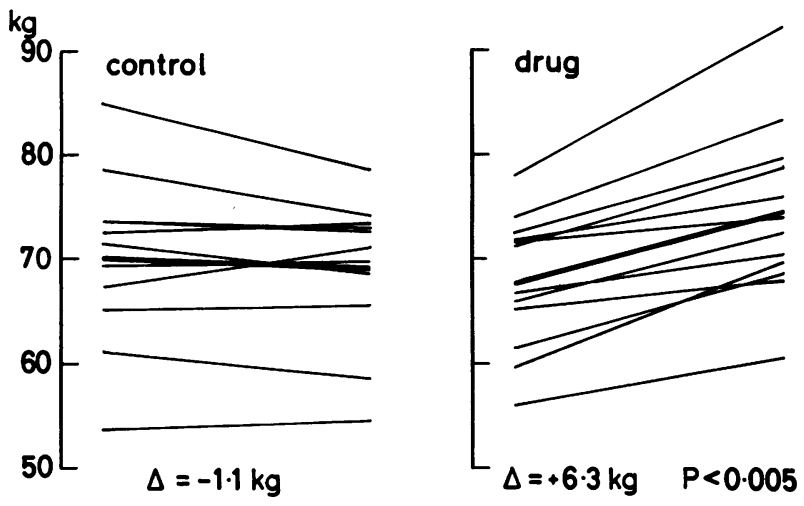

Fig. 2. Changes in the lean body, calculated from measurements of total body potassium, on the basis of a constant concentra: tion of potassium of $66.6 \mathrm{meq} / \mathrm{kg}$ lean tissue.

most of it is within cells, and of these muscle cells are the greater bulk. The body potassium rose on average by $16.4 \mathrm{~g}$ over the treatment period, a significant change, and contrasted with a loss of $2.8 \mathrm{~g}$ over the control period. The best figure for converting potassium to normal lean body mass is probably around $66.6 \mathrm{meq} / \mathrm{kg}$; some uncertainty is unimportant here. This would imply an average increase in lean body mass over the treatment period of $6.3 \mathrm{~kg}$ : substantially more than the change in whole body weight (fig. 2).

It should have been possible also to estimate the change in lean tissue from total body water measured by tritium, but unfortunately some samples were lost as a result of an industrial dispute and others were erratic, 

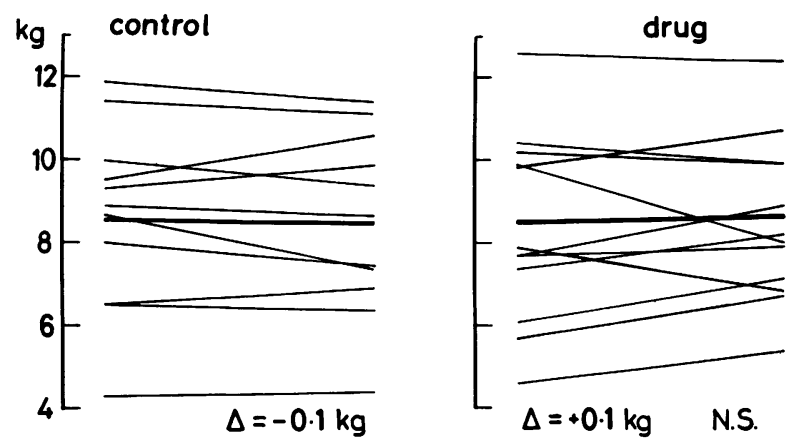

Fig. 3. Changes in body fat, calculated from measurements of skinfolds at four sites (using Durnin \& Rahaman's formula).

perhaps due to fundamental uncertainties in body water methods. Densitometry partitions the body weight into the lean body, assumed to have a density of about 1.1, and fat, with a density of $0.9 \mathrm{~kg} / \mathrm{m}^{3}$. It divided the weight gain over the treatment period into $2.4 \mathrm{~kg}$ lean and $1.0 \mathrm{~kg}$ fat, only the change in lean being statistically significant.

skinfold thickness, measured as the sum of the thickness

Body fat can also be estimated from the correlation with skinfold thickness, measured as the sum of the thickness of folds of skin and subcutaneous tissue measured with "Harpenden" spring-loaded calipers at four sites. The changes found were small and inconsistent (fig. 3). Roentgenogrammetry - X-ray photography with standardized geometry and exposure to show soft tissues - confirmed a significant increase in the width of the thigh muscles on the drug (fig. 4), and a probable increase in the arm muscles. It showed no changes in subcutaneous fat or bone thicknesses.
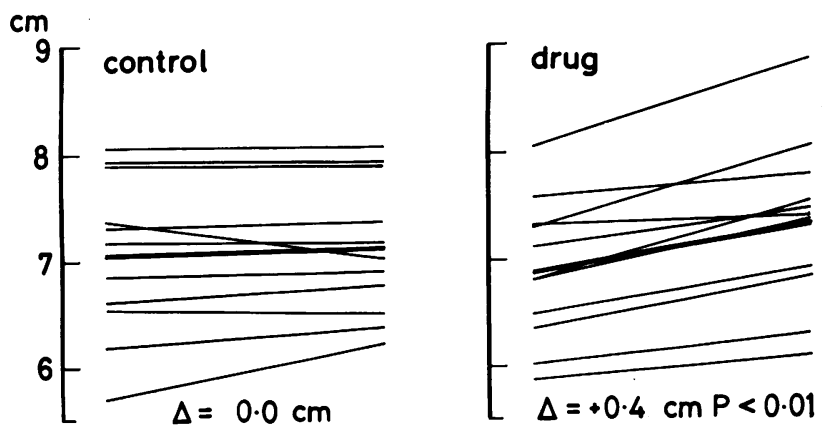

Fig. 4. Changes in width of thigh muscles from Roentgenogrammetry.

Measurements of performance in terms of work and static strength showed a considerable increase in the leg muscles in most subjects over both periods, no doubt as a result of the training regime (fig. 5). (The increases
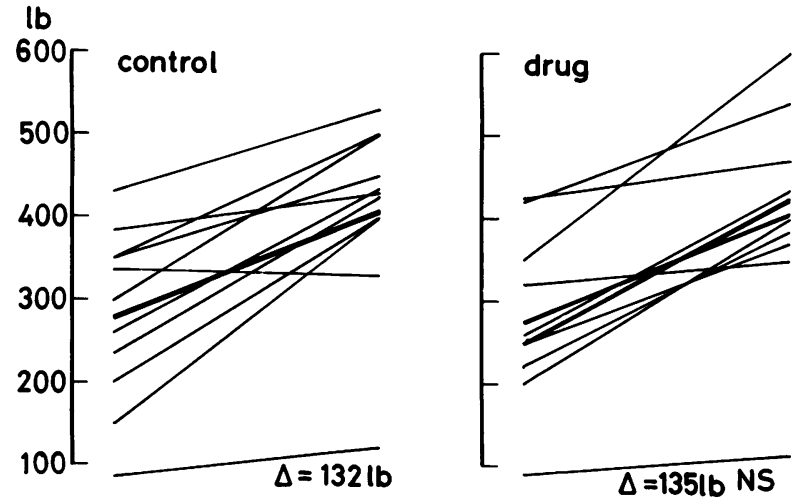

Fig. 5. Changes in "leg press" performance in weight-training.

over the first period disappeared during the holiday). ? There was no evidence that the increases were greater $\rightarrow$ when the drug was being taken. Maximum oxygen up- $\frac{O}{?}$ take showed insignificant increases over both training periods.

The steroid metabolic studies showed that Dianabol $\overrightarrow{0}$ in the dose given suppressed endogenous testosterone ov production, i.e. it acted as an androgen. Clinical and 0 biochemical monitoring of the subjects disclosed no other abnormalities. They completed a questionnaire at the end of the experiment. All had detected when the drug was being given, usually by the change in weight; some reported that they felt more vigour and less fatigue during sport, and one was pleased with the change in his muscle contours.

At first sight the experiment appears to confirm anabolic action. The taking of the steroid led to a consider- $\frac{0}{6}$. able increase in body weight; most and possibly all of 3 this was in the lean body; the muscles increased visibly in size. This would be in contrast with the results of administration to rats. There would, however, still be an $\delta$ open question as to whether the difference in response depended on a species difference, or on the difference in 윽 activity between resting rats and training humans; this might be resolved by experiments using sedentary humans and/or rats somehow induced to exercise. Alter- N natively, perhaps the critical period during development, $c$ at which testosterone can determine that the pattern of $N$ muscle growth as well as other characteristics shall be male, may in man extend later into life, at any rate for muscle growth. (The finding that the male pattern of 0 growth is determined in this way may well be relevant to the problems of presumed female athletes showing some $\stackrel{?}{?}$ degree of intersex).

There is, however, a quite different possibility. Although the significance of negative findings depends $\mathbb{D}$ entirely on their precision, and this can never be high for measurements such as work capacity and strength which 
involve a motivational element, nevertheless some suspicion must be raised by the failure to find any evidence for improvement in performance by muscles which were visibly larger. The scale of the retention of potassium also requires explanation. This must be inside the cells, and possibly the steroid - as a rather non-specific effect at high dose - affects electrolyte transport at cell membranes in such a way as to increase intracellular potassium. Gain of potassium on this scale could not be compensated by loss of sodium from the small amount present in cells, and so water would also enter. Thus, alternatively to the assumption that the increase in muscle size included all constituents including contractile protein - a true anabolic effect - there may be a possibility that it consisted only of water with electrolytes. (This would raise an intriguing analogy with reported practices in the meat trade!)

The experiment thus leaves the question of anabolic action still unsettled. There are, of course, other ways in which athletes may feel benefit from taking "anabolic" steroids. Even if the weight gained is essentially water, it may still be advantageous in throwing events simply because of the mechanics of momentum. The presence of anabolic steroids may increase endogenous corticosteroid levels in stress; attempts were made to test for this, but inconclusively. Androgenic effects on the central nervous system may lead to greater aggressiveness or to insensitivity to pain. There is room for considerably more study, but, if the anabolic action should prove to be a myth, at least one of the major reasons for steroidtaking would be removed.

\section{Acknowledgment}

The investigations described were supported by the Medical Research Council and by Ciba Laboratories. They were carried out by the teams of investigators indicated in the References.

\section{REFERENCES}

Harvey, J. S., Hervey, G. R. and Hutchinson, I. (1972). The role of gonadal hormones in determining the sex difference in adult body weight in the rat. J.Endocr. 57, xxiv.

Hervey, G. R. (1969). Regulation of Energy Balance. Nature, 223, pp 629-631.

Hervey, G. R. and Hutchinson, I. (1972). The effects of testosterone on body weight and composition in the rat. J.Endocr. 57, xxiv-xxv. (A fuller account is in preparation).

Hervey, G. R., Knibbs, A. V., Hutchinson, I., Jones, P. R. M., Burkinshaw, L., Norgan, N. G., Hayter, C. J. and Adamson, G. T. In preparation.

Payne, H. (1975). "Anabolic Steroids in Sport", Brit.Journ.Sports Med., 9: 